\title{
Competencia digital de la inspección educativa en el tratamiento del Big Data
}

\author{
Antonio J. Moreno-Guerrero, Jesús López-Belmonte, Carmen Rodríguez-Jiménez ${ }^{*}$ y Magdalena Ramos \\ Departamento de Didáctica y Organización Escolar, Universidad de Granada, 18071 Granada, España \\ (correo-e: ajmoreno@ugr.es; jesuslopez@ugr.es; carmenrj@ugr.es; magdalenarnp@correo.ugr.es)
}

* Autor a quien debe ser dirigida la correspondencia

Recibido Dic. 10, 2019; Aceptado Feb. 5, 2020; Versión final Mar. 9, 2020, Publicado Jun. 2020

\begin{abstract}
Resumen
El primer objetivo de este estudio es comprobar la relación entre el tratamiento del Big Data y los niveles de competencia digital en una muestra de 386 inspectores educativos. El segundo objetivo es examinar qué implicaciones en el desempeño de su labor profesional podría tener esta relación. Estamos en una época marcada por la tecnología, que está en todos los contextos, siendo la educación donde los procesos y labores derivadas de la praxis educativa se están efectuando en un plano digital, originando un gran volumen de datos. Esto conlleva que entes como la inspección educativa necesite destrezas digitales para un desarrollo profesional eficaz. Se ha utilizado un diseño no experimental y transeccional de tipo descriptivo y correlacional. Los datos se recogieron con un cuestionario ad hoc. Se revela que los inspectores están capacitados para analizar grandes volúmenes de datos. Se concluye que los inspectores tienen un nivel de competencia digital medio, alcanzando buenas destrezas en dos áreas concretas.
\end{abstract}

\section{Digital competences of educational inspection in the treatment of Big Data}

\begin{abstract}
The first objective of this study is to verify the relationship between the treatment of Big Data and the levels of digital competence in a sample of 386 educational inspectors. The second objective is to examine what implications this relationship could have in the performance of their professional jobs. We are in an age marked by technology, which is in all contexts, where educational processes and tasks derived from educational practice are being carried out in a digital plane, originating a great volume of data. This means that entities such as educational inspection need digital skills for effective professional development. A nonexperimental, transactional design of a descriptive and correlational type was used. Data were collected using an ad hoc questionnaire. The results revealed that educational inspectors are capable of analysing large volumes of data. It is concluded that inspectors have a medium level of digital competence, achieving good skills in two specific areas.
\end{abstract}




\section{INTRODUCCIÓN}

Como consecuencia de los constantes avances que suceden a raíz de esta época digital en la que se vive, la tecnología, en su conjunto y en concreto las Tecnologías de la Información y la Comunicación (TIC), de manera particular, tienen una notable presencia e influencia en diferentes sectores de la sociedad, entre los que destaca el educativo, donde esta tecnología juega un papel fundamental, favoreciendo de manera positiva en la mayoría de las ocasiones los procesos formativos de los agentes que actúan dentro de la comunidad educativa (Rodríguez, et al., 2018). Todo esto ha generado que la educación actual desde hace ya algún tiempo esté experimentando una renovación donde todos los elementos y actuaciones están transformándose hacia el desarrollo de nuevos y mejorados contextos donde prima la tecnología, la cual es empleada, entre otras funciones, para la extracción de información que se deriva de estos contextos, así como para su tratamiento y posterior uso (Sánchez-Otero, et al., 2019).

La innovación en la que está inmersa la educación en el momento actual hace necesarias e imprescindibles ciertas habilidades, capacidades y conocimientos, en lo que a tecnología se refiere, para poder desarrollar de manera eficiente todo lo que implica ese proceso innovador que está aconteciendo. Además, es necesario señalar que esto se tiene que dar tanto en docentes como en discentes, pues la familiarización de todos ellos con los diferentes dispositivos y herramientas tecnológicas debe ser amplia, aunque como señala la literatura (Morales, et al., 2016), son los estudiantes los que, por haber nacido y desarrollado en una sociedad digitalizada, tienen generalmente un mayor nivel de adecuación a ellas.

La tecnología educativa pretende entre otros aspectos cubrir las necesidades e intereses de los discentes, por lo que el profesorado tiene que estar capacitado en el manejo eficaz de los dispositivos digitales dentro del aula (Jiménez, et al., 2019; Martínez-Serrano, 2019). Todo esto que se viene plasmando en el presente documento no es un hecho aislado o momentáneo, sino que la vigente ley de educación en el sistema educativo español (Ley Orgánica 8/2013, de 9 de diciembre, para la mejora de la calidad educativa) expone la necesidad y justifica la presencia de las TIC como un elemento que debe aplicarse de un modo pedagógico en todas las áreas del currículo oficial, con el objetivo de extraer el máximo potencial de estos dispositivos y herramientas digitales para que aporten ventajas como pueden ser el aumento de la motivación, tanto de docentes como de discentes, así como la retroalimentación positiva e instantánea, como aspectos más destacados (Liesa, et al, 2016).

Esta implementación y desarrollo de las TIC dentro del aula desde una perspectiva pedagógica ha generado la aparición de experiencias de aprendizaje innovadoras, que tienen como eje central el rol del docente como orientador y guía en el proceso de enseñanza-aprendizaje (PEA) y como agente activo y promovedor del conocimiento, el cual debe estar adaptado y ser personalizado a cada alumno (Fuentes, et al., 2018).

\section{OTROS ANTECEDENTES}

Para este trabajo es conveniente definir algunos conceptos, como los siguientes: la competencia digital de los profesionales de la educación y la generación constante y voluminosa de datos educativos.

\section{La competencia digital de los profesionales de la educación}

La competencia digital es fruto de los avances tecnológicos producidos en la sociedad actual y de los ambientes innovadores surgidos en los espacios de aprendizaje. Por tanto, es entendida como el conjunto de habilidades, destrezas y conocimientos sobre el manejo de la tecnología (Rodríguez-Jiménez, et al., 2019). Dadas las peculiaridades actuales, el nivel de competencia digital de aquellas personas que pertenecen al sector educativo adquiere un matiz relevante en la integración de las TIC en los procesos de PEA desde una perspectiva pedagógica (Jiménez, et al., 2019). Para esto, es necesario la constante formación y actualización de todos los profesionales del ámbito educativo, con el propósito de mejorar las habilidades y capacidades concernientes al uso de los recursos tecnológicos, y para no estar desfasado con respecto a los rápidos cambios y dinamismo que experimenta la tecnología. De este modo, la competencia digital de los diferentes profesionales de la educación es entendida como el conjunto de conocimientos, destrezas y habilidades para el uso de las TIC como elementos integrados en los procesos de PEA (Mañas et al., 2019).

En lo referente a la competencia digital docente existen diversos marcos de referencia tanto a nivel internacional como nacional. Así, en Europa encontramos el DigCompEdu (European Comission, 2017), mientras que en España está el Marco Común de Competencia Digital Docente (INTEF, 2017), el cual está basado en los documentos de la Unión Europea que se acaban de citar. Este marco es una herramienta de autoevaluación para los docentes y está compuesto por cinco áreas competenciales que contienen 21 competencias y que están divididas en seis niveles que se refieren tanto a conocimientos, como habilidades 
y actitudes. Este marco logra identificar a aquellos docentes que son capaces de lograr un manejo e integración eficaz de la tecnología dentro del aula (Rodríguez-Jiménez, et al., 2019). Siguiendo con este marco de referencia, es importante señalar las cinco grandes áreas que lo componen: 1) área de información y alfabetización informacional; 2) área de comunicación y colaboración; 3) área de creación de contenidos digitales; 4) área de seguridad; y 5) área de resolución de problemas.

En este sentido, cabe señalar que no solo se está ante una competencia que es exclusiva de los docentes como agentes que forman parte del contexto educativo, sino que los inspectores educativos son una figura muy a tener en cuenta a este respecto (Moreno, et al., 2020). El papel de estos últimos ha estado siempre marcado por la controversia que genera su regulación, la politización de los mismos o los aspectos administrativos y pedagógicos, pero es innegable los cambios que ha ido sufriendo este organismo en su totalidad con el paso del tiempo. Uno de los cambios más significativos ha sido la adaptación de sus prácticas a causa de la irrupción en la educación de las TIC (Romero, 2018). A pesar de los cambios en la legislación y normas que regulan a estos profesionales de la educación, existe un eje conductor por el que efectúan su praxis y es hacer cumplir la normativa vigente del momento. El servicio que la inspección educativa presta no puede quedar aislado del resto de procesos vivos de los contextos educativos, por eso los progresos y actualizaciones constantes que experimentan los docentes a nivel tecnopedagógico, deberían de experimentarse al mismo nivel y adaptado a su labor los inspectores educativos (Rodríguez, et al., 2018). En los últimos tiempos la inspección educativa está abordando en mayor grado tareas de índole objetiva, esto es, está observando para posteriormente ofrecer a los centros escolares elementos facilitadores como modelos de evaluación entre profesionales y rúbricas, entre otros. Asimismo, la investigación educativa está adentrándose en este sector, como se puede comprobar en la proliferación de estudios recientes alusivos a dicho campo de conocimiento (Moreno, 2019).

Sin embargo, toda esta importancia que se le otorga a la tecnología y a la competencia digital en las políticas educativas del momento, no se ve reflejada en los niveles competenciales que tienen los profesionales de la educación. Diferentes investigaciones en los últimos años (Carrillo, 2018; Fuentes, et al., 2019; Liesa, et al., 2016; Rodríguez-Jiménez, et al., 2019) ponen de manifiesto que el nivel revelado en las diferentes áreas de la competencia digital no es lo suficientemente adecuado como para poder desarrollar de manera eficaz un ejercicio profesional adecuado a una era tecnológica. Todo esto repercute considerablemente en el empleo de las TIC desde una perspectiva tecnopedagógica.

Sin duda, en la actualidad debemos encontrarnos con un cuerpo de profesionales de la educación entre los que se encuentran inspectores educativos y docentes que cuente con la requerida competencia digital entre sus cualidades (Romero, 2018), por lo que es necesario preguntarse las causas de esos bajos niveles en competencia digital que en ocasiones se dan entre ellos. Así, algunas investigaciones han puesto de manifiesto que elementos como las reticencias al cambio y las carencias formativas son las dos principales causas de esta situación La principal finalidad de hoy debe ser reducir esa brecha competencial entre los profesionales de la educación y los estudiantes, quienes estos últimos presentan mejores destrezas (Morales, et al., 2016).

\section{La generación constante y voluminosa de datos educativos}

En los últimos tiempos ha habido un cambio masivo en la capacidad que tenemos de reunir y analizar datos (Mayer-Schönberger, et al., 2018). El Big Data es un fenómeno en el que se unen diversos elementos; por un lado, la tecnología que recopila datos de forma constante; por otro lado, redes que posibilitan el intercambio de esos datos; métodos para la extracción del conocimiento de todos esos datos (ColmenarejoFernández, 2018). En los últimos tiempos, este concepto está siendo ampliamente abordado en la literatura especializada a causa de las interacciones de las personas con los medios digitales, provocando así desmesurados volúmenes de datos (Dishon, 2017). Estos datos y cómo actúan en la sociedad actual dan origen a la denominada segunda era digital, la del Big Data (Pugna, et al., 2019).

Este constructo virtual se alimenta gracias a la conectividad que ha provocado la presencia de la tecnología en la sociedad, en la cual toda actuación de cada persona queda registrada (Huda et al., 2018), pudiendo ser examinada a través de softwares específicos con una amplia capacidad para almacenar, reconocer y analizar bases de datos complejas (Hicks y Irizarry, 2018). El presente interés por investigar sobre el análisis de estos datos radica en conocer lo que los usuarios demandan y, por ende, en la toma de decisiones acertadas para afrontar con eficacia y eficiencia las necesidades de los individuos (Dishon, 2017).

El Big Data se ha extendido por diferentes áreas de conocimiento, siendo la educación una de ellas (Hicks y Irizarry, 2018). Es en este ámbito donde se están recabando grandes cantidades de datos procedentes de los procesos de PEA, derivando esto en un desarrollo docente que se basa en el tratamiento y estudio de 
los datos producidos por los discentes (Gibson, 2017). Es importante señalar que todo esto se enfoca a la mejora de la calidad educativa en lo concerniente a medios virtuales (Liang, et al., 2016) y a la adecuación de las particularidades del estudiantado. Gracias a un análisis exhaustivo del Big Data se propicia la mejora de experiencias que derivan en la innovación educativa, dando lugar así a un amplio abanico de estrategias didácticas en base a esos datos analizados donde poder escoger (Huda et al., 2018).

Sin embargo, para el correcto manejo del Big Data son necesarias determinadas habilidades como: Minería de datos, basado en la transformación de los datos en información útil y significativa (Huda, et al., 2018); Competencia informacional, compuesta por estrategias de búsqueda, selección, evaluación y empleo de los datos (Bielba, et al., 2017); y Pensamiento crítico, para la generación de nuevos datos e información adecuada a los procesos de PEA (Liesa, et al., 2016).

Diferentes estudios exponen que el uso que los docentes hacen del Big Data no llega a tener niveles óptimos de eficacia a causa de las carencias formativas en analítica de datos (Liang et al., 2016). Esta situación deriva en una falta de competencia de los profesionales de la educación en lo concerniente a la gestión, evaluación y transformación de los datos en información. En definitiva, la práctica docente en cuanto al uso y análisis del Big Data se postula como uno de los grandes retos para solucionar las carencias existentes en el contexto educativo, de modo que se consigan mayores niveles de productividad, eficiencia e individualización en los procesos de PEA, adaptado a las particularidades de los discentes a través de la extracción de información relevante después de un exhaustivo análisis de datos educacionales (Dishon, 2017), y específicamente, en el ámbito de la inspección educativa.

\section{MATERIAL Y MÉTODO}

La metodología de investigación empleada en este estudio es de tipo descriptivo y correlacional en base a un diseño de naturaleza no experimental y transeccional, siguiendo las orientaciones de los expertos (Hernández, et al., 2014). El análisis efectuado ha estado compuesto por estadísticos como la media (M), desviación típica (DT), así como diferentes pruebas específicas para determinar la tendencia de la distribución, tales como el coeficiente de asimetría de Pearson (CAP) y el de apuntamiento de Fisher (CAF). Para hallar la relación entre las variables se ha realizado el test Chi-cuadrado de Pearson (x2), acompañado de la V de Cramer (V) y el coeficiente de contingencia (Cont), con el propósito de determinar la fuerza de asociación entre las variables.

\section{Muestra}

Han participado un total de 386 inspectores de educación de diferentes regiones de España. De esta cifra, el $55.44 \%$ son hombres y el resto mujeres, agrupados en los siguientes intervalos de edad: Entre 27 y 35 años= 20.47\%\%; Entre 36 y 45 años=30.05\%; Entre 46 y 55 años=32.12\%; Más de 55 años=17.36\%. En cuanto a la rama de formación en la que desempeñan su labor, el $61.92 \%$ se encuentran en la concerniente a humanidades y ciencias sociales; y el $38.08 \%$ a la conexa a ciencias. La técnica de muestreo para la selección de los participantes ha sido no probabilística. Primeramente, por conveniencia a través de la puesta en contacto con diferentes inspectores de educación de distintas instituciones del territorio español. A su vez, el muestreo fue complementado por la técnica de bola de nieve, solicitando a estos agentes que difundieran el instrumento entre otros profesionales de su mismo cometido.

\section{Instrumento}

Los datos han sido recopilados por medio de un cuestionario ad hoc. Este instrumento ha sido diseñado en base a otras herramientas reportadas de la literatura, alusivas a la valoración de la competencia digital (Agreda, et al., 2016; INTEF, 2017; Instefjord y Munthe, 2017). Concretamente, el cuestionario utilizado aglutina un total de 84 ítems, clasificados en 6 dimensiones: a) Dimensión Socioeducativa (9 ítems); b) Dimensión Información y alfabetización informacional (CD1=12 ítems); c) Dimensión Comunicación y colaboración (CD2=23 ítems); d) Dimensión Creación de contenidos digitales (CD3=14 ítems); e) Dimensión Seguridad (CD4=12 ítems); f) Dimensión Resolución de problemas (CD5=14 ítems). La configuración de las cuestiones se encuentra —en su mayoría - bajo una escala Likert de 4 niveles de puntuación (bajo=BAJ; medio-bajo=MEB; medio-alto=MEA; alto=ALT), así como otras cuestiones que se presentan con respuesta cerrada y dicotómicas.

El proceso de validación del cuestionario se efectuó en primer lugar de manera cualitativa, mediante método Delphi, integrado por 10 especialistas en tecnología educativa de distintas universidades españolas. La valoración otorgada por estos expertos fue adecuada $(M=4.86$, mín=1, max=6; $D T=0.43)$. Asimismo, se aplicaron los estadísticos Kappa de Fleiss y W de Kendall para obtener los índices de conveniencia y similitud de las observaciones de los jueces, cuyos resultados fueron favorecedores $(K=0.84 ; W=0.86)$, centrándose las retroalimentaciones en la mejora de determinados ítems en lo referido a la redacción e interpretación. Seguidamente, el cuestionario fue sometido a un proceso de validación cuantitativa a través de un análisis 
factorial exploratorio mediante el método de componentes principales, utilizando una rotación oblicua. En esta fase, estadísticos como el test de Kaiser-Meyer-Olkin relevó una adecuación muestral $(\mathrm{KMO}=0.81)$ y la prueba de esfericidad de Bartlett obtuvo significancia (7453.64; $\mathrm{gl}=628 ; \mathrm{p}<0.001)$, demostrando dependencia entre las variables establecidas. Por último, se procedió al estudio de la fiabilidad del cuestionario en una muestra independiente de 260 sujetos, empleando diversas medidas de consistencia interna, como el alfa de Cronbach $(\alpha=0.86)$, la fiabilidad compuesta $(F C=0.83)$ y la varianza media extractada (VME $=0.79)$, resultando puntajes que reflejan adecuados índices de fiabilidad de la herramienta de recogida de datos confeccionada para esta investigación.

\section{Variables}

En este apartado se recogen las distintas variables utilizadas en el estudio, junto con su nomenclatura con el fin de mejorar la presentación, visibilidad e interpretación de los resultados: Información y alfabetización informacional (CD1); Comunicación y colaboración (CD2); Creación de contenidos digitales (CD3); Seguridad (CD4); Resolución de problemas (CD5); Navegación, búsqueda, filtrado de información, datos y contenidos digitales (NBFI); Información, datos y contenidos digitales (IDCD); Almacenamiento y recuperación de datos y contenidos digitales (ARDC); Interacción mediante las tecnologías digitales (IMTD); Compartir información y contenidos digitales (CICD); Participación en línea (PCL); Colaboración mediante canales digitales (CMCD); Netiqueta (NETI); Gestión de la identidad digital (GIDI); Desarrollo de contenidos digitales (DCDI); Integración y reelaboración de contenidos digitales (IRCD); Derechos de autor y licencias (DALI); Programación (PROG); Protección de dispositivos (PRDI); Protección de datos personales e identidad digital (PDPE); Protección de la salud (PRSA); Protección del entorno (PREN); Resolución de problemas técnicos (REPT); Identificación de necesidades y respuesta tecnológicas (INRT); Innovación y uso de la tecnología digital de forma creativa (IUTD); Identificación de lagunas en la competencia digital (ILCD); Destreza en el análisis de gran volumen de datos educativos (DADE).

\section{Procedimiento}

El proceso investigador tuvo origen en febrero de 2019 con el diseño y posterior validación del instrumento de recogida de datos. Una vez superada esta fase, se produjo la selección de los participantes mediante las técnicas de muestreo anteriormente descritas. Una vez obtenida la muestra resultante, se produjo el envío del cuestionario por vía telemática (e-mail) en mayo de 2019. El proceso de recogida de datos se dio por finalizado en el mes de junio de 2019, justo antes del periodo vacacional. Una vez recopilados los datos, comenzó la fase de exportación, depuración e importación al programa Statistical Package for the Social Sciences (SPSS) versión 24, permitiendo la elaboración de un análisis en profundidad de las variables que articula este estudio.

\section{RESULTADOS}

En el desarrollo de este apartado se mostrarán los resultados derivados de las variables estudiadas. Tal y como se muestra en la Figura 1, el tratamiento de análisis de grandes volúmenes de datos educativos es una tarea frecuente por parte de los inspectores de educación. Solamente un $5.44 \%$ desarrolla este tipo de acciones de manera escasa. En cambio, casi la mitad de los encuestados (45.34\%) aplican técnicas y acciones encaminada a la búsqueda de datos con una frecuencia muy alta. El resto de sujetos, hacen un uso medio bajo $(21.76 \%)$ y medio alto $(27.46 \%)$.

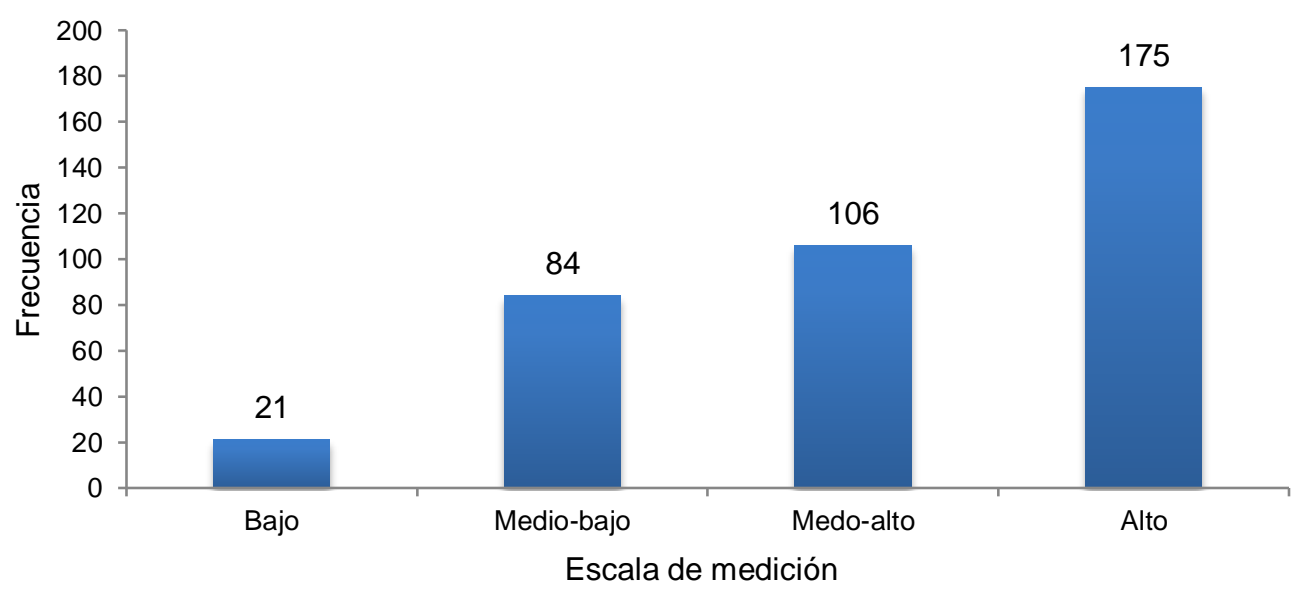

Fig. 1: Destreza en el análisis de gran volumen de datos educativos. 
El nivel de competencia digital, con carácter general, que presentan los inspectores de educación (Tabla 1) es medio, mostrando déficit formativo en determinadas variables, localizadas en todas las áreas competenciales, excepto en la vinculada a la información y alfabetización informacional, donde los valores son medio alto. Los niveles bajos se encuentran localizados en programación e innovación y uso de la tecnología digital de forma creativa, situados en la escala A. En cambio, los niveles más altos se muestran en derechos de autor y licencia, protección de dispositivos y protección de datos personales e identidad personal, donde los resultados son elevados, ubicándose en la escala C. El resto de variables se localizan en la escala B, donde los valores son considerados como medios.

Tabla 1: Descriptivo del nivel de competencia digital por áreas

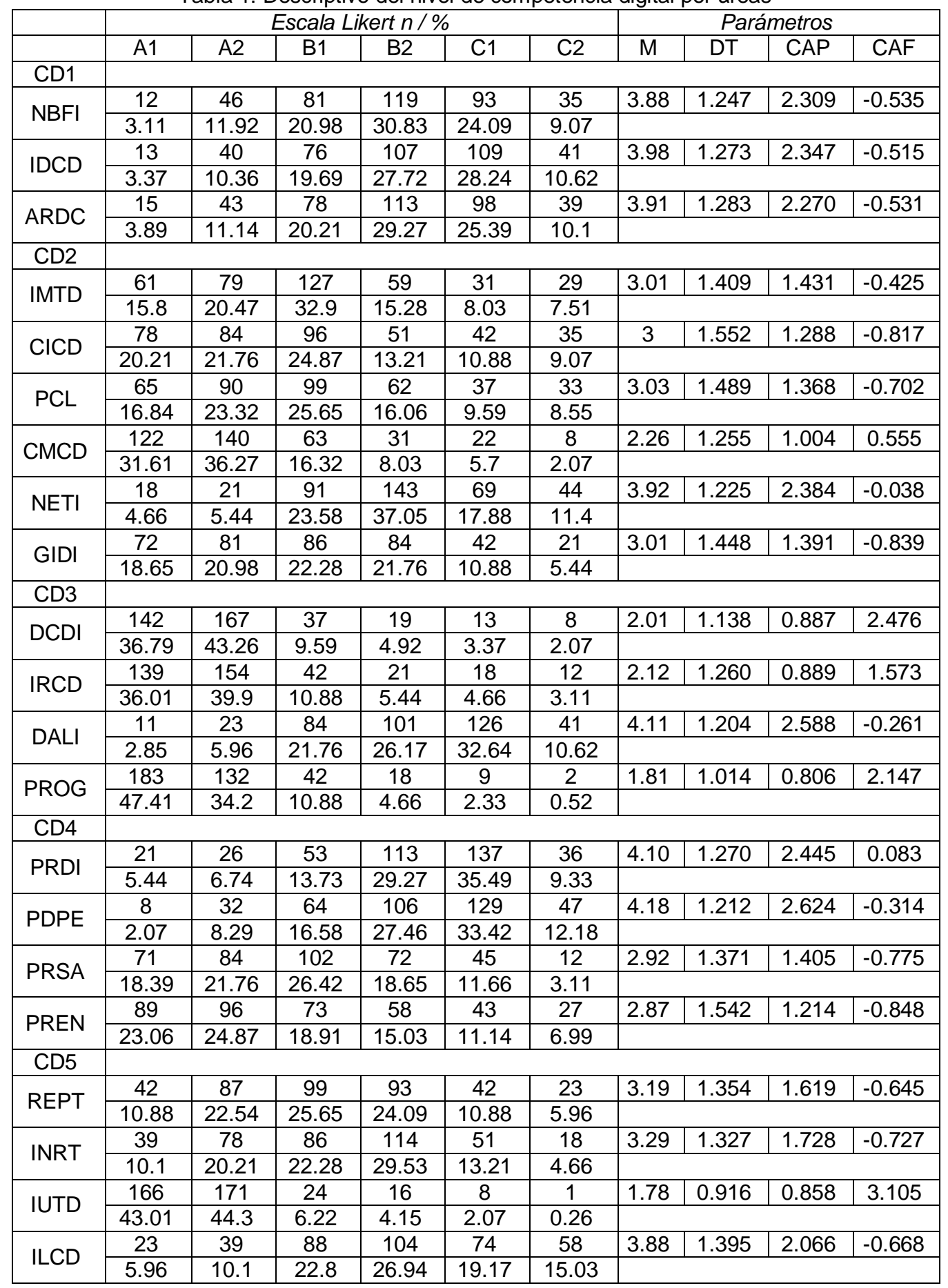

La Figura 2 muestra las medias de las variables analizadas, posicionadas sobre la media ideal y la media totalizada. Como se puede observar, la competencia digital que revelan los inspectores de educación, según la media totalizada, está situada por encima de la media ideal. De manera más específica, hay variables que se alejan de forma considerable de esa media, tanto por encima como por debajo. En este caso, las variables navegación, búsqueda, filtrado de información, datos y contenidos digitales; almacenamiento y recuperación de datos; información, datos y contenidos digitales; netiqueta; derechos de 
autor y licencias; protección de dispositivos; protección de datos personales e identidad personal e identificación de lagunas en la competencia digital, se sitúan cerca de los valores 4 , mientras que las variables colaboración mediante canales digitales; desarrollo de contenidos digitales, integración y reelaboración de contenidos digitales; programación; e innovación y uso de la tecnología digital de forma creativa se ubican cerca de los valores 2 . El resto de variables se sitúan en la media.

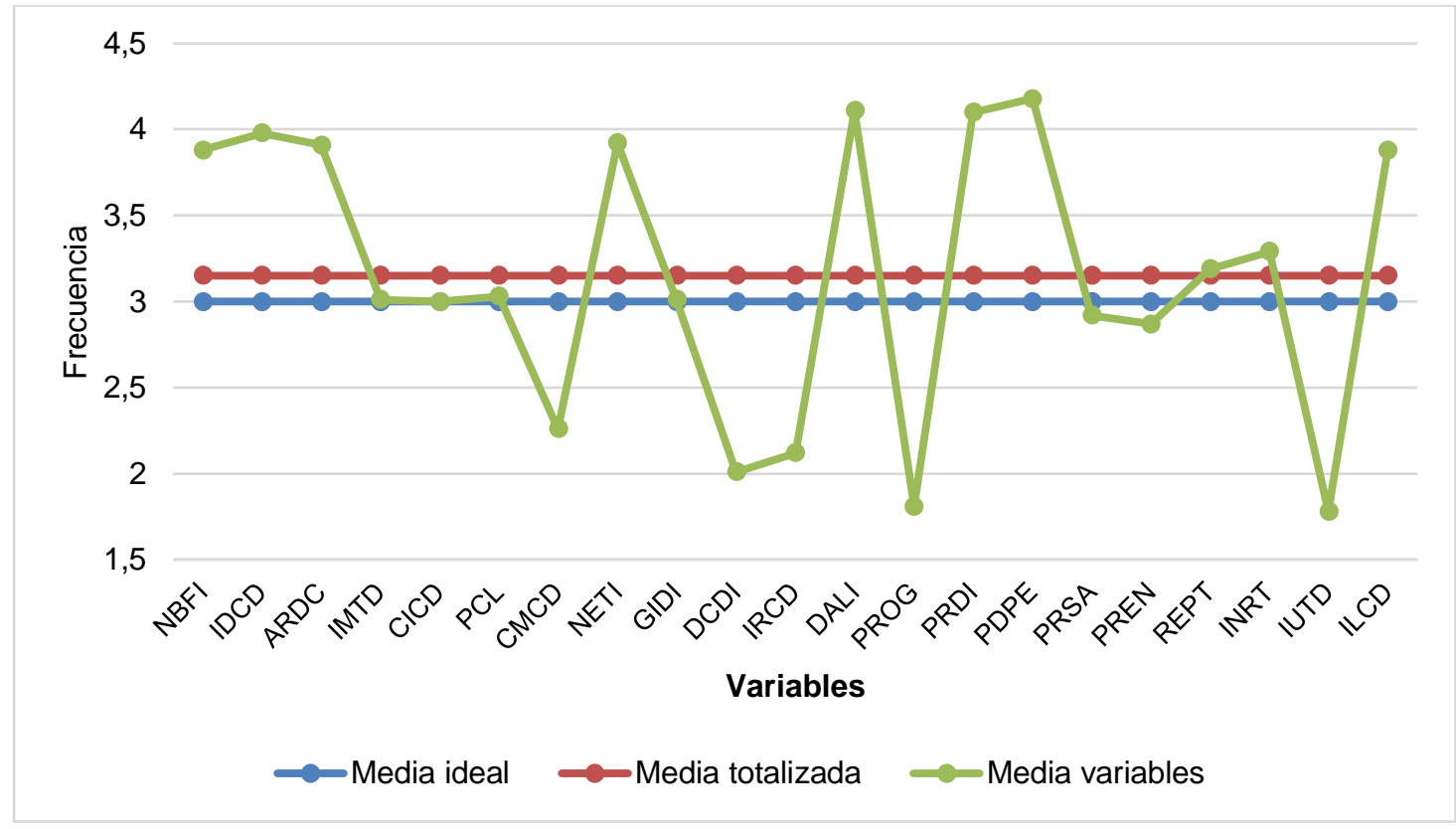

Fig. 2: Distribución de las puntuaciones medias

Tabla 2: Correlación entre CD1 y DADE

\begin{tabular}{|c|c|c|c|c|c|c|c|c|c|c|}
\hline \multirow{2}{*}{ CD1 } & \multicolumn{6}{|c|}{ Escala Likert n / \% } & \multicolumn{4}{|c|}{ Parámetros } \\
\hline & A1 & A2 & $\mathrm{B} 1$ & B2 & C1 & C2 & $x^{2}(g l)$ & $p$-va. & Cont & V \\
\hline NBFI & & & & & & & $76.49(15)$ & $<0.000$ & 0.407 & 0.257 \\
\hline \multirow{2}{*}{ BAJ } & 4 & 2 & 5 & 4 & 5 & 1 & & & & \\
\hline & 1.04 & 0.52 & 1.3 & 1.04 & 1.3 & 0.26 & & & & \\
\hline \multirow{2}{*}{ MEB } & 3 & 20 & 29 & 22 & 5 & 5 & & & & \\
\hline & 0.78 & 5.18 & 7.51 & 5.7 & 1.3 & 1.3 & & & & \\
\hline \multirow{2}{*}{ MEA } & 5 & 12 & 24 & 24 & 33 & 8 & & & & \\
\hline & 1.3 & 3.11 & 6.22 & 6.22 & 8.55 & 2.07 & & & & \\
\hline \multirow{2}{*}{ ALT } & 0 & 12 & 23 & 69 & 50 & 21 & & & & \\
\hline & 0 & 3.11 & 5.96 & 17.88 & 12.95 & 5.44 & & & & \\
\hline IDCD & & & & & & & $114.71(15)$ & $<0.000$ & 0.479 & 0.315 \\
\hline \multirow{2}{*}{ BAJ } & 6 & 7 & 4 & 2 & 1 & 1 & & & & \\
\hline & 1.55 & 1.81 & 1.04 & 0.52 & 0.26 & 0.26 & & & & \\
\hline \multirow{2}{*}{ MEB } & 4 & 16 & 19 & 27 & 13 & 5 & & & & \\
\hline & 1.04 & 4.15 & 4.92 & 6.99 & 3.37 & 1.3 & & & & \\
\hline \multirow{2}{*}{ MEA } & 2 & 14 & 29 & 31 & 21 & 9 & & & & \\
\hline & 0.52 & 3.63 & 7.51 & 8.03 & 5.44 & 2.33 & & & & \\
\hline \multirow{2}{*}{ ALT } & 1 & 3 & 24 & 47 & 74 & 26 & & & & \\
\hline & 0.26 & 0.78 & 6.22 & 12.18 & 19.17 & 6.74 & & & & \\
\hline ARDC & & & & & & & $149.35(15)$ & $<0.000$ & 0.528 & 0.359 \\
\hline \multirow{2}{*}{ BAJ } & 8 & 6 & 3 & 2 & 1 & 1 & & & & \\
\hline & 2.07 & 1.55 & 0.78 & 0.52 & 0.26 & 0.26 & & & & \\
\hline \multirow{2}{*}{ MEB } & 4 & 20 & 18 & 28 & 8 & 6 & & & & \\
\hline & 1.04 & 5.18 & 4.66 & 7.25 & 2.07 & 1.55 & & & & \\
\hline \multirow{2}{*}{ MEA } & 2 & 14 & 31 & 34 & 17 & 8 & & & & \\
\hline & 0.52 & 3.63 & 8.03 & 8.81 & 4.4 & 2.07 & & & & \\
\hline \multirow{2}{*}{ ALT } & 1 & 3 & 26 & 49 & 72 & 24 & & & & \\
\hline & 0.26 & 0.78 & 6.74 & 12.69 & 18.65 & 6.22 & & & & \\
\hline
\end{tabular}

El análisis correlacional de las variables en el área de información y alfabetización digital muestra relación de significancia entre el tratamiento de la información y las tres variables que la conforman. En este caso, la fuerza de relación mostrada en todas ellas es considerada moderada, dado que se ubican en valores situados entre 0.2 y 0.6 según el estadístico V de Cramer (Tabla 2). 
Tabla 3: Correlación entre CD2 y DADE

\begin{tabular}{|c|c|c|c|c|c|c|c|c|c|c|}
\hline \multirow[t]{2}{*}{ CD2 } & \multicolumn{6}{|c|}{ Escala Likert $n / \%$} & \multicolumn{4}{|c|}{ Parámetros } \\
\hline & $\mathrm{A} 1$ & A2 & B1 & B2 & C1 & $\mathrm{C} 2$ & $x^{2}(g l)$ & $p$-va. & Cont & $\mathrm{V}$ \\
\hline IMTD & & & & & & & $47.04(15)$ & $<0.000$ & 0.330 & 0.202 \\
\hline \multirow{2}{*}{ BAJ } & 10 & 4 & 3 & 2 & 1 & 1 & & & & \\
\hline & 2.59 & 1.04 & 0.78 & 0.52 & 0.26 & 0.26 & & & & \\
\hline \multirow{2}{*}{ MEB } & 13 & 22 & 21 & 15 & 8 & 5 & & & & \\
\hline & 3.37 & 5.7 & 5.44 & 3.89 & 2.07 & 1.3 & & & & \\
\hline \multirow{2}{*}{ MEA } & 14 & 18 & 51 & 7 & 3 & 13 & & & & \\
\hline & 3.63 & 4.66 & 13.21 & 1.81 & 0.78 & 3.37 & & & & \\
\hline \multirow{2}{*}{ ALT } & 24 & 35 & 52 & 35 & 19 & 10 & & & & \\
\hline & 6.22 & 9.07 & 13.47 & 9.07 & 4.92 & 2.59 & & & & \\
\hline CICD & & & & & & & $41.41(15)$ & $<0.000$ & 0.311 & 0.189 \\
\hline \multirow{2}{*}{ BAJ } & 9 & 6 & 3 & 2 & 1 & 0 & & & & \\
\hline & 2.33 & 1.55 & 0.78 & 0.52 & 0.26 & 0 & & & & \\
\hline \multirow{2}{*}{ MEB } & 14 & 22 & 21 & 15 & 9 & 3 & & & & \\
\hline & 3.63 & 5.7 & 5.44 & 3.89 & 2.33 & 0.78 & & & & \\
\hline \multirow{2}{*}{ MEA } & 32 & 19 & 31 & 9 & 3 & 12 & & & & \\
\hline & 8.29 & 4.92 & 8.03 & 2.33 & 0.78 & 3.11 & & & & \\
\hline \multirow{2}{*}{ ALT } & 23 & 37 & 41 & 25 & 29 & 20 & & & & \\
\hline & 5.96 & 9.59 & 10.62 & 6.48 & 7.51 & 5.18 & & & & \\
\hline $\mathrm{PCL}$ & & & & & & & $21.18(15)$ & 0.131 & 0.228 & 0.135 \\
\hline \multirow{2}{*}{ BAJ } & 8 & 5 & 3 & 2 & 2 & 1 & & & & \\
\hline & 2.07 & 1.3 & 0.78 & 0.52 & 0.52 & 0.26 & & & & \\
\hline \multirow{2}{*}{ MEB } & 14 & 24 & 19 & 14 & 8 & 5 & & & & \\
\hline & 3.63 & 6.22 & 4.92 & 3.63 & 2.07 & 1.3 & & & & \\
\hline \multirow[b]{2}{*}{ MEA } & 21 & 22 & 35 & 10 & 10 & 8 & & & & \\
\hline & 5.44 & 5.7 & 9.07 & 2.59 & 2.59 & 2.07 & & & & \\
\hline \multirow{2}{*}{ ALT } & 22 & 39 & 42 & 36 & 17 & 19 & & & & \\
\hline & 5.7 & 10.1 & 10.88 & 9.33 & 4.4 & 4.92 & & & & \\
\hline CMCD & & & & & & & $24.95(15)$ & 0.051 & 0.246 & 0.147 \\
\hline \multirow{2}{*}{ BAJ } & 12 & 6 & 2 & 1 & 0 & 0 & & & & \\
\hline & 3.11 & 1.55 & 0.52 & 0.26 & 0 & 0 & & & & \\
\hline & 28 & 25 & 18 & 4 & 9 & 0 & & & & \\
\hline IMEB & 7.25 & 6.48 & 4.66 & 1.04 & 2.33 & 0 & & & & \\
\hline & 24 & 40 & 21 & 12 & 6 & 3 & & & & \\
\hline MEA & 6.22 & 10.36 & 5.44 & 3.11 & 1.55 & 0.78 & & & & \\
\hline AlT & 58 & 69 & 22 & 14 & 7 & 5 & & & & \\
\hline ALI & 15.03 & 17.88 & 5.7 & 3.63 & 1.81 & 1.3 & & & & \\
\hline NETI & & & & & & & $24.64(15)$ & 0.055 & 0.245 & 0.146 \\
\hline & 2 & 5 & 4 & 4 & 4 & 2 & & & & \\
\hline BAJ & 0.52 & 1.3 & 1.04 & 1.04 & 1.04 & 0.52 & & & & \\
\hline MER & 7 & 4 & 18 & 27 & 19 & 9 & & & & \\
\hline IMEB & 1.81 & 1.04 & 4.66 & 6.99 & 4.92 & 2.33 & & & & \\
\hline MFA & 3 & 6 & 25 & 43 & 18 & 11 & & & & \\
\hline TVIEA & 0.78 & 1.55 & 6.48 & 11.14 & 4.66 & 2.85 & & & & \\
\hline & 6 & 6 & 44 & 69 & 28 & 22 & & & & \\
\hline ALT & 1.55 & 1.55 & 11.4 & 17.88 & 7.25 & 5.7 & & & & \\
\hline GIDI & & & & & & & $16.86(15)$ & 0.327 & 0.205 & 0.121 \\
\hline BAI & 2 & 4 & 6 & 5 & 3 & 1 & & & & \\
\hline BAJ & 0.52 & 1.04 & 1.55 & 1.3 & 0.78 & 0.26 & & & & \\
\hline MER & 9 & 24 & 19 & 17 & 10 & 5 & & & & \\
\hline IMEB & 2.33 & 6.22 & 4.92 & 4.4 & 2.59 & 1.3 & & & & \\
\hline MFA & 24 & 27 & 16 & 22 & 10 & 7 & & & & \\
\hline TVIEA & 6.22 & 6.99 & 4.15 & 5.7 & 2.59 & 1.81 & & & & \\
\hline AIT & 37 & 26 & 45 & 40 & 19 & 8 & & & & \\
\hline ALI & 9.59 & 6.74 & 11.66 & 10.36 & 4.92 & 2.07 & & & & \\
\hline
\end{tabular}

En el área competencial de comunicación y colaboración, existen resultados dispares, dado que las variables interacción mediante las tecnologías digitales y compartir información y contenidos digitales ofrecen relación de significancia. En cambio, las variables participación en línea; colaboración mediante canales digitales; netiqueta; y gestión de la identidad digital no se constatan influencia significativa en el cruce de variables. La fuerza de relación de la variable IMTD se considera moderada $(0.2<\mathrm{ES}<0.6)$, mientras que en la variable CICD es baja $(<0.2)$, según el estadístico aplicado (Tabla 3 ). En el área competencial de creación de contenidos digitales, solamente muestra relación de significancia la variable derechos de autor y licencias, cuya fuerza de relación es baja (<.02) según el estadístico aplicado. En el resto de variables no se constata relación de significancia (Tabla 4). 
Tabla 4: Correlación entre CD3 y DADE

\begin{tabular}{|c|c|c|c|c|c|c|c|c|c|c|}
\hline \multirow[t]{2}{*}{ CD3 } & \multicolumn{6}{|c|}{ Escala Likert $n / \%$} & \multicolumn{4}{|c|}{ Parámetros } \\
\hline & A1 & $\mathrm{A} 2$ & B1 & B2 & C1 & $\mathrm{C} 2$ & $x^{2}(g l)$ & $p$-va. & Cont & $\mathrm{V}$ \\
\hline DCDI & & & & & & & $13.87(15)$ & 0.536 & 0.186 & 0.109 \\
\hline \multirow{2}{*}{ BAJ } & 9 & 8 & 3 & 1 & 0 & 0 & & & & \\
\hline & 2.33 & 2.07 & 0.78 & 0.26 & 0 & 0 & & & & \\
\hline \multirow{2}{*}{ MEB } & 32 & 38 & 10 & 4 & 0 & 0 & & & & \\
\hline & 8.29 & 9.84 & 2.59 & 1.04 & 0 & 0 & & & & \\
\hline \multirow{2}{*}{ MEA } & 31 & 52 & 9 & 5 & 5 & 4 & & & & \\
\hline & 8.03 & 13.47 & 2.33 & 1.3 & 1.3 & 1.04 & & & & \\
\hline \multirow{2}{*}{ ALT } & 70 & 69 & 15 & 9 & 8 & 4 & & & & \\
\hline & 18.13 & 17.88 & 3.89 & 2.33 & 2.07 & 1.04 & & & & \\
\hline IRCD & & & & & & & $14.03(15)$ & 0.523 & 0.187 & 0.110 \\
\hline \multirow{2}{*}{ BAJ } & 7 & 7 & 4 & 2 & 1 & 0 & & & & \\
\hline & 1.81 & 1.81 & 1.04 & 0.52 & 0.26 & 0 & & & & \\
\hline \multirow{2}{*}{ MEB } & 31 & 37 & 11 & 2 & 2 & 1 & & & & \\
\hline & 8.03 & 9.59 & 2.85 & 0.52 & 0.52 & 0.26 & & & & \\
\hline \multirow{2}{*}{ MEA } & 30 & 49 & 11 & 6 & 5 & 5 & & & & \\
\hline & 7.77 & 12.69 & 2.85 & 1.55 & 1.3 & 1.3 & & & & \\
\hline \multirow{2}{*}{ ALT } & 71 & 61 & 16 & 11 & 10 & 6 & & & & \\
\hline & 18.39 & 15.8 & 4.15 & 2.85 & 2.59 & 1.55 & & & & \\
\hline DALI & & & & & & & $28.94(15)$ & 0.016 & 0.264 & 0.158 \\
\hline \multirow{2}{*}{ BAJ } & 1 & 5 & 3 & 4 & 3 & 5 & & & & \\
\hline & 0.26 & 1.3 & 0.78 & 1.04 & 0.78 & 1.3 & & & & \\
\hline \multirow{2}{*}{ MEB } & 1 & 6 & 21 & 23 & 24 & 9 & & & & \\
\hline & 0.26 & 1.55 & 5.44 & 5.96 & 6.22 & 2.33 & & & & \\
\hline \multirow{2}{*}{ MEA } & 4 & 7 & 24 & 20 & 38 & 13 & & & & \\
\hline & 1.04 & 1.81 & 6.22 & 5.18 & 9.84 & 3.37 & & & & \\
\hline \multirow{2}{*}{$A L T$} & 5 & 5 & 36 & 54 & 61 & 14 & & & & \\
\hline & 1.3 & 1.3 & 9.33 & 13.99 & 15.8 & 3.63 & & & & \\
\hline PROG & & & & & & & 13.42(15) & 0.570 & 0.183 & 0.108 \\
\hline \multirow{2}{*}{ BAJ } & 8 & 8 & 4 & 1 & 0 & 0 & & & & \\
\hline & 2.07 & 2.07 & 1.04 & 0.26 & 0 & 0 & & & & \\
\hline \multirow{2}{*}{ MEB } & 30 & 36 & 10 & 6 & 1 & 1 & & & & \\
\hline & 7.77 & 9.33 & 2.59 & 1.55 & 0.26 & 0.26 & & & & \\
\hline \multirow{2}{*}{ MEA } & 52 & 34 & 11 & 5 & 3 & 1 & & & & \\
\hline & 13.47 & 8.81 & 2.85 & 1.3 & 0.78 & 0.26 & & & & \\
\hline \multirow{2}{*}{ ALT } & 93 & 54 & 17 & 6 & 5 & 0 & & & & \\
\hline & 24.09 & 13.99 & 4.4 & 1.55 & 1.3 & 0 & & & & \\
\hline
\end{tabular}

En el área competencial de seguridad, todas las variables ofrecen relación de significancia, habiendo fuerza de relación baja con la variable protección de la salud (<.02), y moderada con protección de dispositivos, protección de datos personales e identidad digital y protección del entorno $(0.2<\mathrm{ES}<0.6)$ según los valores hallados por la prueba estadística desarrollada (Tabla 5). Finalmente, en el área competencial conexa a la resolución de problemas, existe relación de significancia entre la variable de destreza en el análisis de gran volumen de datos educativos y el resto de variables que componen esta área. Además, la relación de significancia es moderada, dado que se sitúan en una horquilla intermedia según el estadístico V de Cramer $(0.2<\mathrm{ES}<0.6)$ (Tabla 6$)$.

\section{DISCUSIÓN}

Resulta fundamental adquirir un dominio adecuado de la tecnología pues como se ha postulado anteriormente esta rige a la sociedad actual en todos sus aspectos, siendo la educación uno de los campos de conocimiento donde más influencia ha alcanzado (Rodríguez, et al., 2018). Hacer un uso efectivo y pedagógico de la tecnología en educación facilita el desempeño de la profesión docente y de la inspección educativa. Sin embargo, se encuentran resistencias al cambio y falta de formación por parte de estos profesionales que impiden el buen aprovechamiento de esta herramienta vital en la era digital en la que nos encontramos inmersos (Morales, et al., 2016)

Evaluar la competencia digital es un reto complejo debido a las diferentes dimensiones que la conforman (Lázaro, et al., 2019), las cuales se deben analizar de forma independiente para poder vislumbrar donde se encuentran las mayores carencias y los puntos fuertes de forma concreta. Una vez detectadas dichas necesidades, es más fácil estudiar las formas de mejora que lleven a conseguir el correcto desarrollo de esta competencia esencial para la labor educativa de hoy en día. Investigaciones como la llevada a cabo 
por Agreda, et al. (2016), recogen resultados similares a los obtenidos en este trabajo. En concreto, se encuentran similitudes en los elevados valores alusivos a la información y a la seguridad de forma individual, que en los relacionados con aspectos más innovadores, creativos y colaborativos. Igualmente, otros estudios recientes del mismo modo encuentran diferencias en los distintos aspectos de la competencia digital de los profesionales de la educación, encontrando carencias formativas (Fuentes, et al., 2019; Liesa, et al., 2016; Rodríguez-Jiménez, et al., 2019).

Tabla 5: Correlación entre CD4 y DADE

\begin{tabular}{|c|c|c|c|c|c|c|c|c|c|c|}
\hline \multirow[t]{2}{*}{ CD4 } & \multicolumn{6}{|c|}{ Escala Likert $n$ /\% } & \multicolumn{4}{|c|}{ Parámetros } \\
\hline & A1 & A2 & B1 & B2 & C1 & $\mathrm{C} 2$ & $x^{2}(g l)$ & $p$-va. & Cont & V \\
\hline PRDI & & & & & & & $125.67(15)$ & $<0.000$ & 0.496 & 0.329 \\
\hline \multirow{2}{*}{ BAJ } & 9 & 5 & 1 & 3 & 2 & 1 & & & & \\
\hline & 2.33 & 1.3 & 0.26 & 0.78 & 0.58 & 0.26 & & & & \\
\hline \multirow{2}{*}{ MEB } & 3 & 18 & 14 & 16 & 24 & 9 & & & & \\
\hline & 0.78 & 4.66 & 3.63 & 4.15 & 6.22 & 2.33 & & & & \\
\hline \multirow{2}{*}{ MEA } & 4 & 1 & 14 & 36 & 37 & 14 & & & & \\
\hline & 1.04 & 0.26 & 3.63 & 9.33 & 9.59 & 3.63 & & & & \\
\hline \multirow{2}{*}{ ALT } & 5 & 2 & 24 & 58 & 74 & 12 & & & & \\
\hline & 1.3 & 0.52 & 6.22 & 15.03 & 19.17 & 3.11 & & & & \\
\hline PDPE & & & & & & & $83.03(15)$ & $<0.000$ & 0.421 & 0.268 \\
\hline \multirow{2}{*}{ BAJ } & 2 & 7 & 3 & 2 & 2 & 5 & & & & \\
\hline & 0.52 & 1.81 & 0.78 & 0.52 & 0.52 & 1.3 & & & & \\
\hline \multirow{2}{*}{ MEB } & 2 & 19 & 18 & 13 & 21 & 11 & & & & \\
\hline & 0.52 & 4.92 & 4.66 & 3.37 & 5.44 & 2.85 & & & & \\
\hline \multirow{2}{*}{ MEA } & 3 & 2 & 16 & 34 & 34 & 17 & & & & \\
\hline & 0.78 & 0.52 & 4.15 & 8.81 & 8.81 & 4.4 & & & & \\
\hline \multirow{2}{*}{ ALT } & 1 & 4 & 26 & 57 & 72 & 14 & & & & \\
\hline & 0.26 & 1.04 & 6.74 & 14.77 & 18.65 & 3.63 & & & & \\
\hline PRSA & & & & & & & $29.22(15)$ & 0.015 & 0.265 & 0.159 \\
\hline \multirow{2}{*}{ BAJ } & 7 & 7 & 4 & 1 & 1 & 1 & & & & \\
\hline & 1.81 & 1.81 & 1.04 & 0.26 & 0.26 & 0.26 & & & & \\
\hline \multirow{2}{*}{ MEB } & 18 & 25 & 17 & 13 & 9 & 2 & & & & \\
\hline & 4.66 & 6.48 & 4.4 & 3.37 & 2.33 & 0.52 & & & & \\
\hline \multirow{2}{*}{ MEA } & 23 & 15 & 35 & 14 & 13 & 6 & & & & \\
\hline & 5.96 & 3.89 & 9.07 & 3.63 & 3.37 & 1.55 & & & & \\
\hline \multirow{2}{*}{ ALT } & 23 & 37 & 46 & 44 & 22 & 3 & & & & \\
\hline & 5.96 & 9.59 & 11.92 & 11.4 & 5.7 & 0.78 & & & & \\
\hline PREN & & & & & & & $46.79(15)$ & $<0.000$ & 0.329 & 0.201 \\
\hline \multirow[b]{2}{*}{ BAJ } & 10 & 6 & 5 & 0 & 0 & 0 & & & & \\
\hline & 2.59 & 1.55 & 1.3 & 0 & 0 & 0 & & & & \\
\hline \multirow{2}{*}{ MEB } & 21 & 32 & 12 & 4 & 7 & 8 & & & & \\
\hline & 5.44 & 8.29 & 3.11 & 1.04 & 1.81 & 2.07 & & & & \\
\hline \multirow[b]{2}{*}{ MEA } & 24 & 29 & 21 & 11 & 15 & 6 & & & & \\
\hline & 6.22 & 7.51 & 5.44 & 2.85 & 3.89 & 1.55 & & & & \\
\hline \multirow[b]{2}{*}{ ALT } & 34 & 29 & 35 & 43 & 21 & 13 & & & & \\
\hline & 8.81 & 7.51 & 9.07 & 11.14 & 5.44 & 3.37 & & & & \\
\hline
\end{tabular}

\section{DISCUSION FINAL}

Particularmente, en el presente estudio, dentro de los niveles más altos de competencia digital que poseen los inspectores educativos destacan los aspectos referidos a los derechos de autor y licencia, protección de dispositivos y protección de datos personales e identidad personal. De forma general, se demuestra que la competencia digital que poseen los inspectores de educación se sitúa por encima de la media ideal. Sin embargo, hay variables que se encuentran muy por debajo de esta como son: la colaboración mediante canales digitales, el desarrollo de contenidos digitales, integración y reelaboración de contenidos digitales, programación e innovación y uso de la tecnología digital de forma creativa, todos ellos necesarios para el asesoramiento pedagógico en los centros educativos. Por otro lado, las variables navegación, búsqueda, 
filtrado de información, datos y contenidos digitales, almacenamiento y recuperación de datos, información, datos y contenidos digitales, netiqueta, derechos de autor y licencias, protección de dispositivos, protección de datos personales e identidad personal e identificación de lagunas en la competencia digital, se sitúan dentro de los valores que superan esta media ideal.

Al asociar los niveles de destrezas de los inspectores de educación con el análisis de un gran volumen de datos educativos en cada una de las áreas de la competencia digital estudiadas, se muestra que existe una relación significativa entre el tratamiento de la información y las tres variables que contiene el área conexa a la información y alfabetización informacional: navegación, búsqueda y filtrado de información; información, datos y contenidos digitales y almacenamiento y recuperación de datos y contenidos digitales. Igual sucede con el área competencial de comunicación y colaboración: las variables interacción mediante las tecnologías digitales y compartir información y contenidos digitales muestran también una relación de significancia. En el área competencial de creación de contenidos digitales, solamente se ha encontrado una relación de significancia con la variable derechos de autor y licencias. Así mismo, dentro del área competencial de seguridad se comprueba que todas las variables poseen una relación significativa. $Y$ en lo que se refiere al área competencial resolución de problemas, existe una relación significativa moderada entre la variable destreza en el análisis de gran volumen de datos educativos y el resto de variables que la conforman.

Tabla 6: Correlación entre CD5 y DADE

\begin{tabular}{|c|c|c|c|c|c|c|c|c|c|c|}
\hline \multirow[t]{2}{*}{ CD5 } & \multicolumn{6}{|c|}{ Escala Likert $n / \%$} & \multicolumn{4}{|c|}{ Parámetros } \\
\hline & A1 & A2 & B1 & B2 & C1 & $\mathrm{C} 2$ & $x^{2}(g l)$ & $p$-va. & Cont & $\mathrm{V}$ \\
\hline REPT & & & & & & & $57.72(15)$ & $<0.000$ & 0.361 & 0.223 \\
\hline \multirow{2}{*}{ BAJ } & 9 & 7 & 2 & 2 & 0 & 1 & & & & \\
\hline & 2.33 & 1.81 & 0.52 & 0.52 & 0 & 0.26 & & & & \\
\hline \multirow{2}{*}{ MEB } & 9 & 31 & 17 & 14 & 7 & 6 & & & & \\
\hline & 2.33 & 8.03 & 4.4 & 3.63 & 1.81 & 1.55 & & & & \\
\hline \multirow{2}{*}{ MEA } & 11 & 28 & 22 & 25 & 13 & 7 & & & & \\
\hline & 2.85 & 7.28 & 5.7 & 6.48 & 3.37 & 1.81 & & & & \\
\hline \multirow{2}{*}{ ALT } & 13 & 21 & 58 & 52 & 22 & 9 & & & & \\
\hline & 3.37 & 5.44 & 15.03 & 13.47 & 5.7 & 2.33 & & & & \\
\hline INRT & & & & & & & 49.04(15) & $<0.000$ & 0.336 & 0.206 \\
\hline \multirow{2}{*}{ BAJ } & 8 & 8 & 3 & 1 & 1 & 0 & & & & \\
\hline & 2.07 & 2.07 & 0.78 & 0.26 & 0.26 & 0 & & & & \\
\hline \multirow{2}{*}{ MEB } & 7 & 26 & 15 & 24 & 6 & 6 & & & & \\
\hline & 1.81 & 6.74 & 3.89 & 6.22 & 1.55 & 1.55 & & & & \\
\hline \multirow{2}{*}{ MEA } & 9 & 24 & 21 & 35 & 13 & 4 & & & & \\
\hline & 2.33 & 6.22 & 5.44 & 9.07 & 3.37 & 1.04 & & & & \\
\hline \multirow{2}{*}{ ALT } & 15 & 20 & 47 & 54 & 31 & 8 & & & & \\
\hline & 3.89 & 5.18 & 12.18 & 13.99 & 8.03 & 2.07 & & & & \\
\hline IUTD & & & & & & & $62.67(15)$ & $<0.000$ & 0.374 & 0.233 \\
\hline \multirow{2}{*}{ BAJ } & 15 & 1 & 4 & 0 & 0 & 1 & & & & \\
\hline & 3.89 & 0.26 & 1.04 & 0 & 0 & 0.26 & & & & \\
\hline \multirow{2}{*}{ MEB } & 46 & 31 & 4 & 2 & 1 & 0 & & & & \\
\hline & 11.92 & 8.03 & 1.04 & 0.52 & 0.26 & 0 & & & & \\
\hline \multirow{2}{*}{ MEA } & 49 & 45 & 10 & 1 & 1 & 0 & & & & \\
\hline & 12.69 & 11.66 & 2.59 & 0.26 & 0.26 & 0 & & & & \\
\hline \multirow{2}{*}{ ALT } & 56 & 94 & 6 & 13 & 6 & 0 & & & & \\
\hline & 14.51 & 24.35 & 1.55 & 3.37 & 1.55 & 0 & & & & \\
\hline ILCD & & & & & & & $58.15(15)$ & $<0.000$ & 0.362 & 0.224 \\
\hline \multirow{2}{*}{ BAJ } & 5 & 4 & 4 & 3 & 2 & 3 & & & & \\
\hline & 1.3 & 1.04 & 1.04 & 0.78 & 0.52 & 0.78 & & & & \\
\hline \multirow{2}{*}{ MEB } & 5 & 14 & 18 & 13 & 16 & 18 & & & & \\
\hline & 1.3 & 3.63 & 4.66 & 3.37 & 4.15 & 4.66 & & & & \\
\hline \multirow{2}{*}{ MEA } & 11 & 14 & 19 & 21 & 25 & 16 & & & & \\
\hline & 2.85 & 3.63 & 4.92 & 5.44 & 6.48 & 4.15 & & & & \\
\hline \multirow{2}{*}{$\mathrm{ALT}$} & 2 & 7 & 47 & 67 & 31 & 21 & & & & \\
\hline & 0.52 & 0.78 & 12.18 & 17.36 & 8.03 & 5.44 & & & & \\
\hline
\end{tabular}

A la vista de los resultados obtenidos en esta investigación se ha podido analizar la relación existente entre el tratamiento del Big Data y los niveles de competencia digital por parte de la inspección educativa, pudiéndose concluir que los inspectores de educación están familiarizados con el análisis de grandes cantidades de datos, dados los resultados obtenidos en este estudio, además poseen un nivel en la competencia digital medio, presentando déficit en su formación en algunas variables de todas las áreas competenciales estudiadas, como en programación e innovación y uso de la tecnología digital de forma 
creativa. Solamente en lo que se refiere a la información y alfabetización informacional se ha obtenido un nivel de competencia digital medio alto. Dada la importancia de adquirir una competencia digital adecuada por parte de los inspectores de educación para el óptimo desempeño de su labor, queda evidente que todavía existen lagunas que suplir en cuestiones formativas, situándose por debajo de del nivel óptimo en los que se debe hacer hincapié. Esto confirma la necesidad de una formación continua que permita estar al día con la tecnología dinámica que caracteriza y va unidad a nuestra sociedad.

La prospectiva de la investigación se centra en ofrecer a las Administraciones educativas competentes un perfil de la inspección educativa en relación a la competencia digital docente y su relación para el tratamiento del Big Data. Con respecto a las limitaciones, se han encontrado dificultades a la hora de que los inspectores de educación participantes rellenaran los cuestionarios enviados vía correo electrónico, dado que, al realizarlos de forma online, se pierde una cantidad de la muestra que por falta de tiempo u olvido no contesta los ítems, además de necesitar de una insistencia constante y continua para obtener la muestra que se ha presentado en este estudio. Otro aspecto a destacar en las limitaciones es la muestra utilizada, dado que se ha usado una muestra por conveniencia, no usando técnicas de muestreo específico, por lo que se debe tener cuidado a la hora de extrapolar estos resultados a toda la población. Como futura línea de investigación, se propone analizar el uso y manejo de programas informáticos específicos para el tratamiento y análisis de los big data en el ámbito educativo.

\section{CONCLUSIONES}

De acuerdo al trabajo presentado y a los resultados obtenidos, se pueden plantear las siguientes conclusiones principales:

1.- Los inspectores de educación presentan niveles de competencia digital alto en aspectos directamente relacionados con su trabajo, centrados principalmente en aspectos normativos y legislativos, en los que destacan los derechos de autor, el uso de licencias, la protección de dispositivos, la protección de datos personales y la identidad personal, en cambio muestran niveles bajos en aspectos relacionados con acciones pedagógicas en el uso de las tecnologías de la información y comunicación, como puede ser la colaboración mediantes canales digitales, el desarrollo de contenidos digitales, la integración y reelaboración de contenidos digitales, programación de recursos pedagógicos, innovación pedagógica y uso de la tecnología digital de forma creativa.

2.- Existe una estrecha relación entre el tratamiento de la información, y todas las áreas competenciales, en especial con la seguridad, lo que muestra que el uso del Big Data por la inspección educativa requiere, para su adecuado manejo y uso, un nivel adecuado de competencia digital en todas las áreas competenciales.

3.- Los inspectores de educación muestran destrezas digitales adecuadas para el uso y tratamiento de grandes datos, lo que facilita su trabajo diario, como puede ser la supervisión y análisis de documentos institucionales o el estudio y evaluación de datos económicos y organizativos.

\section{REFERENCIAS}

Agreda, M., M.A. Hinojo y J.M. Sola, Design and validation of an instrument for assess Digital Skills of teachers in Spanish Higher Education, Píxel-Bit: Revista de Medios y Educación, 49, 39-56, (2016).

Bielba, M., F. Martínez y M.J. Rodríguez, Validación psicométrica de un instrumento de evaluación de Competencias Informacionales en la Educación Secundaria, Bordón. Revista de pedagogía, 49(1), 27-43, (2017).

Carrillo, E., School supervision: Impacts, dilemmas and transformation in basic education, Revista de Investigación Educativa de la Escuela de Graduados en Educación, 8(16), 30-39, (2018).

Colmenarejo-Fernández, R. Ética aplicada a la gestión de datos masivos. Anales de la Cátedra Francisco Suárez, (52), 113-129, (2018).

European Comission., DigCompEdu. Digital Competence for Educators. EU Science Hub (2017).

Fuentes, A., J. López y S. Pozo, Análisis de la Competencia Digital Docente: Factor clave en el desempeño de pedagogías activas con Realidad Aumentada, REICE: Revista Iberoamericana sobre Calidad, Eficacia y Cambio en Educación, 17(2), 27-42, (2019).

Ghani, N., S. Hamid, I. Targio y E. Ahmed, Social media Big data analytics: A survey, Computers in Human Behavior, 1, 1-12, (2018).

Gibson, D., Big data in Higher Education: research methods and analytics supporting the learning journey, Technology, Knowledge and Learning, 12(3), 237-241, (2017).

Gobierno de España (2013), Ley Orgánica 8/2013, de 9 de diciembre, para la mejora de la calidad educativa. (BOE núm. 295: 1-64), Madrid, 10 de diciembre de 2013. 
Hernández, R., C. Fernández y M.P. Baptista, Metodología de la investigación, McGraw-Hill Education, México, D.F. (2014).

Hicks, S. y R. Irizarry, A guide to teaching data science, The American, 72(4), 382-391, (2018).

Huda, M., A. Maseleno, y otros cinco autores, Big data emerging technology: insights into Innovative Environment for online learning resources, International Journal of Emerging Technologies in Learning (iJET), 13(1), 23-36, (2018).

Instefjord, E. y E. Munthe, Educating digitally competent teachers: A study of integration of Professional Digital Competence in teacher education, Teaching and teacher education, 67, 37-45, (2017).

INTEF, Instituto Nacional de Tecnologías Educativas y de Formación del Profesorado (2017), Marco Común de Competencia Digital Docente

Jiménez, D., P. Sancho y S. Sánchez, Perfil del futuro docente: Nuevos retos en el marco de EEES, Contextos Educativos. Revista de Educación, 23, 125-139, (2019).

Lázaro, J., M. Usart y M. Gisbert, Assessing Teacher Digital Competence: the Construction of an Instrument for Measuring the Knowledge of Pre-Service Teachers, Journal of New Approaches In Educational Research2, 8(1), 73-78, (2019).

Liang, J., J. Yang, y otros tres autores, Big data application in education: dropout prediction in edx MOOCs. In, 2016 IEEE Second International Conference on Multimedia Big Data (BigMM), pp. 440-443, (2016).

Liesa, M., S. Vázquez y J. Lloret, Identifying the strengths and weaknesses of the digital competency in the use of internet applications in first grade of the teacher degree, Revista Complutense De Educación, 27(2), 845-862, (2016).

Mañas, A., y R. Roig-Vila. Las Tecnologías de la Información y la Comunicación en el ámbito educativo. Un tándem necesario en el contexto de la sociedad actual. Revista Internacional d'Humanitats, (45), 75-86, (2019).

Martínez-Serrano, M. C. Percepción de la Integración y uso de las Tecnologías de la Información y la Comunicación (TIC). Estudio de Profesores y Estudiantes de Educación Primaria. Información tecnológica, 30(1), 237-246, (2019).

Mayer-Schönberger, V., y E. Ingelsson. Big Data and medicine: a big deal?. Journal of internal medicine, 283(5), 418429, (2018).

Morales, M., J.M. Trujillo y F. Raso, Perceptions of Teachers and students to the university potential of ITC integration in the educational process: thoughts, experiences and research in the Faculty of Education of Granada, Edmetic, 5(1), 113142, (2016).

Moreno, A.J., Estudio bibliométrico de la producción científica sobre la Inspección Educativa, REICE. Revista Iberoamericana sobre Calidad, Eficacia y Cambio en Educación, 17(3), 23-40, (2019).

Moreno, A.J., J.M. Romero, M. Ramos y S. Alonso, Análisis bibliométrico sobre Inspección Educativa en la base de datos Web of Science, REICE. Revista Iberoamericana Sobre Calidad, Eficacia y Cambio En Educación, 18(1), 83-103, (2020).

Pugna, I., A. Duțescu y O. Stanila, Corporate Attitudes towards Big Data and Its Impact on Performance Management: $A$ Qualitative Study, Sustainability, 11(3), 1-26, (2019).

Rodríguez, A., M.P. Cáceres y Santiago Alonso, La Competencia Digital del futuro docente: Análisis Bibliométrico de la productividad científica indexada en Scopus, International Journal of Educational Research and Innovation. IJERI, 10, 317-333, (2018).

Rodríguez-Jiménez, C., J.M. Romero-Rodríguez y N. Campos-Soto, La Competencia Digital de los Futuros Docentes. Un reto de la Educación Superior. In Redes-Innovaestic, (Edited by A. Lledó-Carreras, and J.M. Antolí-Martínez. Alicante: Universidad de Alicante, pp. 216-229, (2019).

Rodríguez, M., C. Álvarez y A. Camacho, Claves para el diseño de un plan de formación permanente para la Inspección Educativa en España, Avances en Supervisión Educativa, 29, 60- 78, (2019).

Romero, M. Use of ICT by educational inspection in Andalusia, EDMETIC, 7(1), 275-296, (2018).

Sánchez-Otero, M., J. García-Guiliany, E. Steffens-Sanabria, y H. H. Palma, Estrategias Pedagógicas en Procesos de Enseñanza y Aprendizaje en la Educación Superior incluyendo Tecnologías de la Información y las Comunicaciones. Información tecnológica, 30(3), 277-286, (2019). 
